\title{
Flow Structure Identification for Nonlinear Dynamical Systems via Finite-Time Lyapunov Analysis
}

\author{
M. Maggia ${ }^{1}$, K.D. Mease ${ }^{1 *}$ \\ ${ }^{1}$ Department of Mechanical and Aerospace Engineering, University of California Irvine \\ CA, 92697, USA
}

\begin{abstract}
Identifying and characterizing geometric structure in the flow of a nonlinear dynamical system can facilitate understanding, model simplification, and solution approximation. The approach addressed in this paper uses information from finite-time Lyapunov exponents and vectors associated with the tangent linear dynamics. We refer to this approach as finitetime Lyapunov analysis (FTLA). FTLA identifies the potential for flow structure based on the stability and timescales implied by the spectrum of finite-time Lyapunov exponents. The corresponding Lyapunov vectors provide a basis for representing a splitting of the tangent space at phase points consistent with the splitting of the spectrum. A key property that makes FTLA viable is the exponential convergence of the splitting as the finite time increases. Tangency conditions for the vector field are used to determine points on manifolds of interest. The benefits of the FTLA approach are the dynamical model need not be in a special normal form, the manifolds of interest need not be attracting nor of known dimension, and the manifolds need not be associated with a fixed point or periodic orbit.

After a brief review of FTLA, it is applied to spacecraft stationkeeping around a libration point in the circular restricted three-body problem. This application requires locating the stable and unstable subspaces at points on periodic and aperiodic orbits. For the periodic case, the FTLA subspaces are shown to agree with the Floquet subspaces; for the quasi-periodic case, the accuracy of the FTLA subspaces is demonstrated by simulation.
\end{abstract}

Keywords and phrases: finite-time Lyapunov exponents, finite-time Lyapunov vectors, flow structure, nonlinear dynamical systems, timescales, circular restricted three-body problem, spacecraft stationkeeping

Mathematics Subject Classification: 34D08, 34D35, 34N05, 37D05, 37D25, 37D30, $37 \mathrm{~J} 15,37 \mathrm{M} 25$

\section{Introduction}

Knowledge of geometric structure in the flow of a dynamical system can benefit understanding, model reduction, and solution approximation. As an example, two-timescale behavior in a finite-dimensional dynamical system may be associated with reduced-order manifold structure. If the dynamical system is

${ }^{*}$ Corresponding author. E-mail: kmease@uci.edu

(C) EDP Sciences, 2015 
linear and time-invariant, the eigenvalues of the system matrix characterize the timescales in a coordinateindependent manner. If there exist modes that decay rapidly relative to the time interval of interest, then the system trajectories quickly approach a reduced-order manifold, in this case a reduced-order linear subspace that is the product of the eigenspaces associated with the 'slower' eigenvalues. The (generalized) eigenvectors associated with the slower eigenvalues serve as a basis for the reduced-order linear subspace. For a nonlinear time-invariant system, such eigenanalysis is applicable to the linearized dynamics at an equilibrium point and can provide a local approximation to a two-timescale induced reduced-order manifold. For a periodic orbit of a nonlinear system, eigenanalysis of the monodromy matrix for the linearized dynamics can provide a local approximation to a two-timescale induced reduced-order manifold. For some systems, such as Hamiltonian systems, there can be fast unstable as well as stable behavior in addition to slower behavior. We refer to this as partially hyperbolic structure in reference to the rigorous asymptotic theory in [17]. Associated with partially hyperbolic structure there may exist a reduced-order center manifold; if so, it is normally hyperbolic.

How does one determine geometric structure in the flow of a nonlinear system away from equilibria and periodic orbits? See [6] for an overview of the methods that have been developed. We mention in particular the pseudo steady-state approximation [28], singular perturbations [25,26], the intrinsic lowdimensional manifold (ILDM) method [22], the computational singular perturbation (CSP) method [21], an invariance partial differential equation approach [27], and the zero derivative principle [12]. These methods have been successful when applicable. They do have limitations such as requiring a priori the appropriate normal form for the system equations (all but ILDM and CSP), requiring significant timescale separation and limited manifold curvature (ILDM), and requiring a normally attracting reduced-order manifold (CSP). The theory of partially hyperbolic sets $[3,17]$ does not have these limitations, but its asymptotic nature and hypotheses are not appropriate for many situations of practical interest. The theory of partially hyperbolic sets in the nonuniform case is based on asymptotic Lyapunov exponents [3]. Considering instead finite-time Lyapunov exponents (FTLEs) extends the applicability of the concepts from the theory of partially hyperbolic sets. Unlike the asymptotic case, the finite-time information is metric dependent; but it does allow flow structure to be resolved that would not be sensed asymptotically.

In this paper we first briefly review finite-time Lyapunov analysis (FTLA) following [24]; other versions can be found in $[1,5,11,16,29,35]$ and the references therein. The version of FTLA in [24] is patterned after the asymptotic theory of partially hyperbolic sets with important differences. It relies more heavily on the finite-time Lyapunov vectors and associated subspaces, because the convergence rate (toward the ideal asymptotic counterparts) for this information is much higher than that for the finite-time Lyapunov exponents. Characterizing the convergence rate leads to a guideline for how much finite-time is required. The novel contribution of the present paper is to apply this version of FTLA to orbits and manifold structure in the circular restricted three-body problem (CR3BP) for purposes motivated by spacecraft stationkeeping. Overviews of the application area, and in particular the dynamical systems approach that has been pursued, can be found in $[4,15,19]$. For stationkeeping, Floquet theory has been used to obtain local approximations to the manifold structure near an orbit. When the orbit is periodic, this is appropriate. Floquet theory has also been applied for near-periodic orbits. We propose FTLA as an alternative means of determining manifold structure, one that does not require periodicity, though it has its own requirements which will be clarified. Finite-time Lyapunov vectors (FTLVs) are used to identify the stable and unstable tangent subspaces that can serve as local approximations to the associated stable and unstable manifolds. The accuracy of the stable and unstable FTLVs is verified by comparison to the corresponding Floquet vectors for periodic orbits about the $L_{1}$ libration point in the Earth-Moon circular restricted 3-body problem. Then the accuracy of the stable and unstable FTLVs is demonstrated by forward and backward simulation, respectively, for points on quasi-periodic $L_{1}$ orbits. We note that FTLE fields are used in [30] for the same purpose. 


\section{Finite-Time Lyapunov Analysis of Nonlinear Dynamical Systems}

Finite-time Lyapunov analysis is applied to a given coordinate representation of an autonomous finitedimensional nonlinear dynamical system of the form

$$
\dot{\mathbf{x}}=\mathbf{f}(\mathbf{x}),
$$

with phase vector of coordinates $\mathbf{x} \in \mathbb{R}^{n}$ in the standard basis, the vector field $\mathbf{f}$ a smooth vector-valued function, and $\dot{\mathbf{x}}:=d \mathbf{x} / d t$ with $t$ denoting the independent variable time. The solution of (2.1) for the initial phase point $\mathbf{x}$ is denoted by $\mathbf{x}(t)=\phi(t, \mathbf{x})$, where $\phi(t, \cdot): \mathbb{R}^{n} \rightarrow \mathbb{R}^{n}$ is the $t$-dependent flow map associated with the vector field $\mathbf{f}$ and the initial condition is $\phi(0, \mathbf{x})=\mathbf{x}$.

The tangent linear dynamics associated with (2.1) are

$$
\dot{\mathbf{v}}=D \mathbf{f}(\mathbf{x}) \mathbf{v}
$$

where $\mathbf{v} \in T_{\mathbf{x}} \mathbb{R}^{n}$. Let $\Phi(t, \mathbf{x})$ denote the transition matrix defined as the fundamental matrix for tangent linear dynamics initialized with the identity matrix calculated from a point $\mathbf{x}$, i.e., $\Phi=\partial \phi / \partial \mathbf{x}$.

In FTLA, the tangent linear dynamics are analyzed to diagnose timescales and stability and characterize the modal subspaces of the tangent space at a point $\mathbf{x}$ on a generic orbit, i.e., not necessarily an equilibrium point or a point on a periodic orbit. The characteristic exponential rates and associated directions are provided by, respectively, the finite-time Lyapunov exponents (FTLEs) and finite-time Lyapunov vectors (FTLVs). For a vector $\mathbf{v} \in T_{\mathbf{x}} \mathbb{R}^{n}$, the forward and backward FTLEs are defined by

$$
\mu^{ \pm}(T, \mathbf{x}, \mathbf{v}):=\frac{1}{T} \ln \frac{\|\Phi( \pm T, \mathbf{x}) \mathbf{v}\|}{\|\mathbf{v}\|}
$$

where $T$ is the propagation time, also referred to as the averaging time, and is always taken to be positive whether the propagation is forward or backward. Variables computed by forward and backward propagation are labeled with superscripts ${ }^{+}$and ${ }^{-}$respectively. The $\operatorname{FTLVs~}_{i}^{+}(T, \mathbf{x}), i=1, \ldots, n$ are an orthonormal basis of $T_{\mathbf{x}} \mathbb{R}^{n}$ with the minimum sum of exponents, i.e., the minimum value of $\sum_{i=1}^{n} \mu_{i}^{+}\left(T, \mathbf{x}, \mathbf{l}_{i}^{+}(T, \mathbf{x})\right)$ over all orthonormal bases [7]. The forward Lyapunov spectrum is the set of exponents corresponding to the minimizing solution, namely, $\left\{\mu_{i}^{+}(T, \mathbf{x}), i=1, \ldots, n\right\}$. For a given $(T, \mathbf{x})$, the Lyapunov spectrum is unique, though the minimizing basis is not in general. One way [7] to obtain a minimizing basis (FTLVs) and the forward Lyapunov spectrum (FTLEs) is to compute the singular value decomposition (SVD) of

$$
\Phi(T, \mathbf{x})=N^{+}(T, \mathbf{x}) \Sigma^{+}(T, \mathbf{x}) L^{+}(T, \mathbf{x})^{T},
$$

where

$$
\Sigma^{+}(T, \mathbf{x})=\operatorname{diag}\left(\sigma_{1}^{+}(T, \mathbf{x}), \ldots, \sigma_{n}^{+}(T, \mathbf{x})\right),
$$

contains the singular values, all positive and assumed ordered such that $\sigma_{1}^{+}(T, \mathbf{x}) \leq \sigma_{2}^{+}(T, \mathbf{x}) \leq \cdots \leq$ $\sigma_{n}^{+}(T, \mathbf{x})$, and to compute the FTLEs as $\mu_{i}^{+}(T, \mathbf{x})=(1 / T) \ln \sigma_{i}^{+}(T, \mathbf{x}), i=1, \ldots, n$. The column vectors of the matrix $L^{+}(T, \mathbf{x})$ are the minimizing orthonormal basis vectors $\mathbf{l}_{i}^{+}(T, \mathbf{x}), i=1, \ldots, n$ for $T_{\mathbf{x}} \mathbb{R}^{n}$, and the column vectors of the orthogonal matrix $N^{+}(T, \mathbf{x})$, denoted $\mathbf{n}_{i}^{+}(T, \mathbf{x}), i=1, \ldots, n$, provide a basis for $T_{\phi(T, \mathbf{x})} \mathbb{R}^{n}$. Similarly, the backward exponents can be obtained from the singular value decomposition

$$
\Phi(-T, \mathbf{x})=N^{-}(T, \mathbf{x}) \Sigma^{-}(T, \mathbf{x}) L^{-}(T, \mathbf{x})^{T},
$$

where

$$
\Sigma^{-}(T, \mathbf{x})=\operatorname{diag}\left(\sigma_{1}^{-}(T, \mathbf{x}), \ldots, \sigma_{n}^{-}(T, \mathbf{x})\right) .
$$

We assume the ordering on the diagonal of $\Sigma^{-}(T, \mathbf{x})$ is such that $\sigma_{1}^{-}(T, \mathbf{x}) \geq \cdots \geq \sigma_{n}^{-}(T, p)$. The FTLEs in the backward Lyapunov spectrum are $\mu_{i}^{-}(T, \mathbf{x})=(1 / T) \ln \sigma_{i}^{-}(T, \mathbf{x}), i=1, \ldots, n$. The column vectors 
of the orthogonal matrix $L^{-}(T, \mathbf{x})$ are denoted by $\mathbf{l}_{i}^{-}(T, \mathbf{x}), i=1, \ldots, n$. For the column vectors of $L^{-}(T, \mathbf{x})$ and the orthogonal matrix $N^{-}(T, \mathbf{x})$, we have $\mathbf{l}_{i}^{-}(T, \mathbf{x}) \in T_{\mathbf{x}} \mathbb{R}^{n}$ whereas $\mathbf{n}_{i}^{-}(T, \mathbf{x}) \in T_{\phi(-T, \mathbf{x})} \mathbb{R}^{n}$. The $\mathbf{l}_{i}^{+}(T, \mathbf{x})$ and the $\mathbf{l}_{i}^{-}(T, \mathbf{x})$ vectors, for $i=1, \ldots, n$, are referred to as forward and backward FTLVs, respectively.

The orthonormal finite-time Lyapunov vectors $\mathbf{l}_{i}^{ \pm}(T, \mathbf{x})$ for $i=1, \ldots, n$, define the finite-time Lyapunov subspaces

$$
\begin{aligned}
& \mathcal{L}_{i}^{+}(T, \mathbf{x}):=\operatorname{span}\left\{\mathbf{l}_{1}^{+}(T, \mathbf{x}), \ldots, \mathbf{l}_{i}^{+}(T, \mathbf{x})\right\} \\
& \mathcal{L}_{i}^{-}(T, \mathbf{x}):=\operatorname{span}\left\{\mathbf{l}_{i}^{-}(T, \mathbf{x}), \ldots, \mathbf{l}_{n}^{-}(T, \mathbf{x})\right\} .
\end{aligned}
$$

\subsection{Finite-Time Partially Hyperbolic Spectra and Tangent Space Splittings}

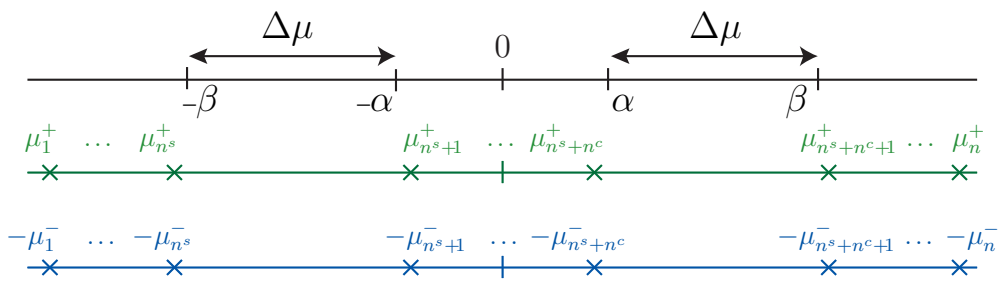

FiguRE 1. Spectra of forward and backward Lyapunov exponents in forward and backward time illustrating a partially hyperbolic spectrum, the constants $\alpha$ and $\beta$ bounding the stable, center, and unstable spectral subsets, and the spectral gap $\Delta \mu$. The forward (resp. backward) exponents are indicated by "+" (resp. "-") superscripts.

Definition 2.1. A point $\mathrm{x} \in \mathbb{R}^{n}, n \geq 2$, is uniform finite-time partially hyperbolic for (2.1) with respect to the Euclidean metric, with fast time constant $\nu^{-1}$ and slow time constant $\sigma^{-1}$, and convergence time constant $\Delta \mu^{-1}$, resolvable over $\Delta \mu\left(\bar{T}-t_{s}\right)$ convergence time constants, if there exist non-negative integers $n^{s}, n^{c}$ and $n^{u}$, with $n^{s}+n^{c}+n^{u}=n, n^{c} \geq 1$ and $n^{s}+n^{u} \geq 1$, a delayed start time $t_{s}$, a cut-off time $t_{c}$, and an available averaging time $\bar{T}$ with $0 \leq t_{s}<t_{c} \leq \bar{T}$ such that the following three properties are satisfied. We use the notation $\mathcal{T}=\left(t_{s}, \bar{T}\right]$ and $\mathcal{T}_{c}=\left(t_{s}, t_{c}\right]$.

1. Uniform Spectral Gaps - There exist positive constants $\alpha$ and $\beta$ with $\beta-\alpha>0$ such that, uniformly on $\mathcal{T}$, the forward and backward FTLE spectra are separated by gaps of size $\Delta \mu=\beta-\alpha$ into $n^{s}, n^{c}$ and $n^{u}$ dimensional subsets as illustrated in Figure 1 and specified by

$$
\begin{aligned}
\mu_{n^{s}}^{+} & \leq-\beta, \quad-\alpha \leq \mu_{n^{s}+1}^{+}, \quad \mu_{n^{s}+n^{c}}^{+} \leq \alpha, \quad \beta \leq \mu_{n^{s}+n^{c}+1}^{+} \\
-\mu_{n^{s}}^{-} & \leq-\beta, \quad-\alpha \leq-\mu_{n^{s}+1}^{-}, \quad-\mu_{n^{s}+n^{c}}^{-} \leq \alpha, \quad \beta \leq-\mu_{n^{s}+n^{c}+1}^{-} .
\end{aligned}
$$

2. Tangent Space Splitting - For each averaging time $T \in \mathcal{T}$, there is a splitting

$$
T_{\mathbf{x}} \mathbb{R}^{n}=\mathcal{E}^{s}(T, \mathbf{x}) \oplus \mathcal{E}^{c}(T, \mathbf{x}) \oplus \mathcal{E}^{u}(T, \mathbf{x}),
$$

where

$$
\begin{aligned}
& \mathcal{E}^{s}(T, \mathbf{x})=\mathcal{L}_{n^{s}}^{+}(T, \mathbf{x}), \\
& \mathcal{E}^{c}(T, \mathbf{x})=\mathcal{L}_{n^{s}+n^{c}}^{+}(T, \mathbf{x}) \cap \mathcal{L}_{n^{s}+1}^{-}(T, \mathbf{x}), \\
& \mathcal{E}^{u}(T, \mathbf{x})=\mathcal{L}_{n^{s}+n^{c}+1}^{-}(T, \mathbf{x}) .
\end{aligned}
$$


3. Two Timescales - There exist positive numbers $\nu$ and $\sigma$ with $\nu>\sigma$ such that for all $t \in \mathcal{T}_{c}$

$$
\begin{aligned}
& \mathbf{v} \in \mathcal{E}^{s}(\bar{T}, \mathbf{x}) \Rightarrow\left\{\begin{array}{l}
\|\Phi(-t, \mathbf{x}) \mathbf{v}\| \geq e^{\nu t}\|\mathbf{v}\| \\
\|\Phi(t, \mathbf{x}) \mathbf{v}\| \leq e^{-\nu t}\|\mathbf{v}\|
\end{array}\right. \\
& \mathbf{v} \in \mathcal{E}^{c}(\bar{T}, \mathbf{x}) \Rightarrow\left\{\begin{array}{l}
e^{-\sigma t}\|\mathbf{v}\| \leq\|\Phi(t, \mathbf{x}) \mathbf{v}\| \leq e^{\sigma t}\|\mathbf{v}\| \\
e^{-\sigma t}\|\mathbf{v}\| \leq\|\Phi(-t, \mathbf{x}) \mathbf{v}\| \leq e^{\sigma t}\|\mathbf{v}\|
\end{array}\right. \\
& \mathbf{v} \in \mathcal{E}^{u}(\bar{T}, \mathbf{x}) \Rightarrow\left\{\begin{array}{l}
\|\Phi(-t, \mathbf{x}) \mathbf{v}\| \leq e^{-\nu t}\|\mathbf{v}\| \\
\|\Phi(t, \mathbf{x}) \mathbf{v}\| \geq e^{\nu t}\|\mathbf{v}\|
\end{array}\right.
\end{aligned}
$$

The properties are stated for the general case where $n^{s}$ and $n^{u}$ are both nonzero. Either $n^{s}$ or $n^{u}$ can be zero, but not both. For $n^{s}=0, \mathcal{E}^{s}$ is not relevant; similarly, for $n^{u}=0, \mathcal{E}^{u}$ is not relevant.

This definition of a uniform finite-time partially hyperbolic point is adapted from [24]. In that reference the definition is for a two-timescale set of points in the phase space for which the required properties are uniformly satisfied. Here we state it for a single point. When Def. 2.1 is applied point-wise, the constants will in general be $\mathbf{x}$-dependent. The term 'two-timescale' refers to the fact that exponential rates in $\mathcal{E}^{c}(\bar{T}, \mathbf{x})$ are slow relative to those in $\mathcal{E}^{s}(\bar{T}, \mathbf{x})$ and $\mathcal{E}^{u}(\bar{T}, \mathbf{x})$. For a finite-time partially hyperbolic point $\mathbf{x}$, the structure of the forward and backward FTLE spectra, as in Figure 1, are required on an interval of averaging times $\left[t_{s}, \bar{T}\right]$ where $t_{s}>0$ is a start time that excludes the initial transient period $\left[0, t_{s}\right]$ during which 'non-modal behavior' can influence the FTLEs. For flow structure to be present over a finite duration of time $t_{f}$, the fast time constant $\nu^{-1}$ should be small relative to $t_{f}$ so that the vectors in $\mathcal{E}^{s}$ and $\mathcal{E}^{u}$ decay quickly in forward and backward time respectively with respect to $t_{f}$. In contrast the vectors in $\mathcal{E}^{c}$ should not decay or grow significantly in forward or backward time. The cut-off time $t_{c}$ avoids a potential final transient from $t_{c}$ to $\bar{T}$ when a subspace rotates away from the ideal asymptotic subspace it is intended to approximate.

The finite-time Lyapunov subspaces converge towards fixed subspaces at a rate at least proportional to $e^{-\Delta \mu T}$ and so do the subspaces $\mathcal{E}^{s}(T, \mathbf{x}), \mathcal{E}^{c}(T, \mathbf{x})$, and $\mathcal{E}^{u}(T, \mathbf{x})$. In order for the finite-time subspaces to approximate their ideal infinite-time limits accurately, the product $\Delta \mu\left(\bar{T}-t_{s}\right)$ should be at least 5 , as a guideline.

The convergence of $\mathcal{E}^{s}$ is discussed in more detail next. The details for $\mathcal{E}^{c}$ and $\mathcal{E}^{u}$ are similar. We say that $\mathcal{E}^{s}(T, \mathbf{x})$ converges toward a fixed subspace and denote this fixed subspace by $\mathcal{E}^{s}(\mathbf{x})$. We characterize the rate of convergence of $\mathcal{E}^{s}(T, \mathbf{x})$ toward the ideal $\mathcal{E}^{s}(\mathbf{x})$ using a time-uniform lower bound on the spectral gap between $\mu_{n^{s}}^{+}$and $\mu_{n^{s}+1}^{+}$over an interval of averaging times $\left(t_{s}, \bar{T}\right]$. By ideal, we mean that convergence in principle is only achieved asymptotically if the spectral gap persists. An accurate finitetime approximation of the ideal subspace may be feasible, because of the exponential rate of convergence discussed next.

Definition 2.2. Over the time interval $\left(t_{s}, \bar{T}\right]$, the time-uniform lower bound on the spectral gap between $\mu_{n^{s}}^{+}(T, \mathbf{x})$ and $\mu_{n^{s}+1}^{+}(T, \mathbf{x})$ is $\Delta \mu^{+}(\mathbf{x})=\inf _{T \in\left(t_{s}, \bar{T}\right]}\left(\mu_{n^{s}+1}^{+}(T, \mathbf{x})-\mu_{n^{s}}^{+}(T, \mathbf{x})\right)$.

Convergence is quantified as the decreasing distance between the finite-time subspace $\mathcal{E}^{s}(T, \mathbf{x})$ and the subspace $\mathcal{E}^{s}(T+\Delta T, \mathbf{x})$ as the averaging time $T$ increases, where $\Delta T$ is a fixed increment to $T$. The distance between $\mathcal{S}_{1}$ and $\mathcal{S}_{2}$, subspaces of $\mathbb{R}^{n}$ of same dimension, is given by [13]

$$
\operatorname{dist}\left(\mathcal{S}_{1}, \mathcal{S}_{2}\right):=\left\|P_{1}-P_{2}\right\|_{2},
$$

where $P_{1}$ and $P_{2}$ are orthogonal projections on $\mathcal{S}_{1}$ and $\mathcal{S}_{2}$ respectively, and $\|\cdot\|_{2}$ is the induced 2-norm. The distance has a value in the interval $[0,1]$. The largest principal angle $\theta \in[0, \pi / 2]$ between the subspaces is given by $\sin \theta=\operatorname{dist}\left(\mathcal{S}_{1}, \mathcal{S}_{2}\right)$. Let

$$
\begin{aligned}
L_{n^{s}}^{+}(T, \mathbf{x}) & :=\left[\mathbf{l}_{1}^{+}(T, \mathbf{x}) \cdots \mathbf{l}_{n^{s}}^{+}(T, \mathbf{x})\right], \\
{\left[L_{n^{s}}^{+}(T, \mathbf{x})\right]^{\perp} } & :=\left[\mathbf{l}_{n^{s}+1}^{+}(T, \mathbf{x}) \cdots \mathbf{l}_{n}^{+}(T, \mathbf{x})\right] .
\end{aligned}
$$


Note that the column space of matrix $L_{n^{s}}^{+}$is the subspace $\mathcal{L}_{n^{s}}^{+}$and the column span of $L_{n^{s}}^{\perp}$ is the orthogonal complement of $\mathcal{L}_{n}^{+}$. In [24], it is shown that the distance between the subspaces $\mathcal{E}^{s}(T, \mathbf{x})$ and $\mathcal{E}^{s}(T+\Delta T, \mathbf{x})$ is given by

$$
\operatorname{dist}\left(\mathcal{E}^{s}(T, \mathbf{x}), \mathcal{E}^{s}(T+\Delta T, \mathbf{x})\right)=\left\|L_{n^{s}}^{+}(T, \mathbf{x})^{T} L_{n^{s}}^{\perp}(T+\Delta T, \mathbf{x})\right\|_{2} .
$$

and that given a spectral gap bound $\Delta \mu^{+}(\mathbf{x})$ at $\mathbf{x} \in \mathcal{W}^{s}$, the subspace $\mathcal{E}^{s}(T, \mathbf{x})$ approaches a fixed subspace $\mathcal{E}^{s}(\mathbf{x})$ at an exponential rate characterized, for every sufficiently small $\Delta T>0$, by the existence of a constants $C>0$ and $T_{1}>t_{s}$ such that

$$
\operatorname{dist}\left(\mathcal{E}^{s}(T, \mathbf{x}), \mathcal{E}^{s}(T+\Delta T, \mathbf{x})\right) \leq C e^{-\Delta \mu^{+}(\mathbf{x}) T}
$$

for $T>T_{1}$.

\subsection{Manifold Conditions}

When FTLA has diagnosed partially hyperbolic structure in a region of phase space, we can postulate a corresponding manifold structure for the flow of the nonlinear system (2.1). In the idealized case in which both the splitting $T_{\mathbf{x}} \mathcal{X}=\mathcal{E}^{s}(\mathbf{x}) \oplus \mathcal{E}^{c}(\mathbf{x}) \oplus \mathcal{E}^{u}(\mathbf{x})$ and the center $\mathcal{W}^{c}$, center-stable $\mathcal{W}^{c s}$, and center-unstable $\mathcal{W}^{c u}$ manifolds are invariant, at points on the manifolds, the tangency conditions

$$
\begin{gathered}
\mathbf{x} \in \mathcal{W}^{c} \Rightarrow \mathbf{f}(\mathbf{x}) \in \mathcal{E}^{c}(\mathbf{x}) \\
\mathbf{x} \in \mathcal{W}^{c s} \Rightarrow \mathbf{f}(\mathbf{x}) \in \mathcal{E}^{s}(\mathbf{x}) \oplus \mathcal{E}^{c}(\mathbf{x}) \\
\mathbf{x} \in \mathcal{W}^{c u} \Rightarrow \mathbf{f}(\mathbf{x}) \in \mathcal{E}^{c}(\mathbf{x}) \oplus \mathcal{E}^{u}(\mathbf{x}) .
\end{gathered}
$$

are satisfied.

Approximating the invariant splitting by the non-invariant finite-time version (2.10), points on approximations of the invariant manifolds can be calculated by applying finite-time tangency conditions in form of orthogonality conditions. For example, the manifold $\mathcal{W}^{c}(\bar{T})$ is a $n^{c}$-dimensional submanifold and represents the finite-time approximation of the invariant normally hyperbolic center manifold $\mathcal{W}^{c}$. The points in the set

$$
\left\{\mathbf{x} \in \mathcal{X}:\langle\mathbf{f}(\mathbf{x}), \mathbf{w}\rangle=0, \forall \mathbf{w} \in\left[\mathcal{E}^{c}(\bar{T}, \mathbf{x})\right]^{\perp}\right\}
$$

satisfy necessary conditions for being on the finite-time center manifold $\mathcal{W}^{c}(\bar{T})$. It can be shown that the finite-time Lyapunov vectors

$$
\mathbf{l}_{1}^{-}(\bar{T}, \mathbf{x}), \ldots, \mathbf{l}_{n^{s}}^{-}(\bar{T}, \mathbf{x}), \mathbf{l}_{n^{s}+n^{c}+1}^{+}(\bar{T}, \mathbf{x}), \ldots, \mathbf{l}_{n}^{+}(\bar{T}, \mathbf{x})
$$

form a basis for $\left[\mathcal{E}^{c}(\bar{T}, \mathbf{x})\right]^{\perp}$. In a similar fashion, we can find approximations of points on the center-stable $\mathcal{W}^{c s}(\bar{T})$ and center-unstable $\mathcal{W}^{c u}(\bar{T})$ manifolds. For further details see [24].

It is important to note that the timescales indicated by the FTLEs apply to the flow of the tangent linear dynamics. The motion on $\mathcal{W}^{c}$ need not be slow in an absolute sense.

\section{Application to Spacecraft Maneuvers}

Dynamical systems theory has proven especially useful in spacecraft mission design and operations when the spacecraft dynamics model considers more than one additional gravitational body; see for example [4, 20] and the references therein. In particular the model for the circular restricted 3-body problem (CR3BP) has been useful for conceptualization of spacecraft maneuvers and to provide a first-approximation of the dynamics and orbit structure for more precise multi-body models. The 5 equilibrium points in the synodic (rotating) frame, called libration points and denoted $L_{1}, \ldots, L_{5}$, are useful spacecraft destinations for scientific purposes. Several past and future space missions targeting orbits around such points have been designed including ISEE-3, launched in 1978, SOHO in 1995, ARTEMIS in 2001, and the future James 
Webb Space Telescope which is scheduled to launch in 2018 and aims to place the large telescope into orbit about the (Earth+Moon)-Sun $L_{2}$ libration point.

FTLA has a potential role regarding spacecraft maneuvers as we shall demonstrate in the context of orbits about the $L_{1}$ libration point in the Earth-Moon-spacecraft CR3BP. In the neighborhood of $L_{1}$, there are periodic orbits, including Lyapunov horizontal and halo orbits, and also quasi-periodic orbits $[2,8]$. Generically each of these orbits has associated stable and unstable manifolds, whose fibers are locally approximated at each point on the orbit by stable and unstable transverse one-dimensional subspaces. Thus the orbits around $L_{1}$ are unstable. A spacecraft intended to follow an $L_{1}$ orbit will drift away from $L_{1}$ due to perturbations. Maintaining an $L_{1}$ orbit requires repeated propulsive maneuvers, and this maneuvering is called stationkeeping.

The natural dynamics can be exploited for the design of efficient transfers to and from orbits around $L_{1}$. For example, if a spacecraft is inserted on the stable manifold of one of these orbits, it will, in principle, reach the orbit without using propulsion [4,20]. For insertion into and stationkeeping about $L_{1}$ orbits, required capabilities include determining stable and unstable directions in the tangent space at points in phase space. In [10] it is shown that for stationkeeping the fuel-minimizing thrust impulse direction is well-approximated by the stable direction. In [31] a stationkeeping approach of using a thrust impulse to cancel the unstable component of the phase error is described. The methods $[4,14,15,18]$ that have been used to approximate the manifold structure for $L_{1}$ orbits have required periodicity or nearperiodicity of the orbit. This is because the local approximations of the stable and unstable manifolds are computed via Floquet analysis; the appropriate eigenvectors of the monodromy matrix are used to approximate the stable and unstable subspaces. For near-periodic orbits an approximate period must be chosen. Finite-time Lyapunov analysis does not require periodicity or near-periodicity of the orbit, thus it offers the potential for analyzing more complicated orbits associated with multi-body gravitational models.

\subsection{CR3BP Spacecraft Dynamics and Libration Points}

The three-body problem concerns the motions of three bodies with masses $m_{1}, m_{2}$, and $m_{3}$ that are coupled due to mutual gravitational forces. The Circular Restricted Three-Body Problem (CR3BP) refers to a three-body problem in which $m_{3}$, the secondary mass, has negligible gravitational influence on the other two masses $m_{1}$ and $m_{2}$, the primaries, but is influenced by the gravity of $m_{1}$ and $m_{2}$, and each primary is in a circular orbit about the center of mass (barycenter) of primaries. For further background on the CR3BP see [32].

We consider in particular the Earth-Moon-spacecraft CR3BP with $m_{1}$ the Earth mass, $m_{2}$ the Moon mass, and $m_{3}$ the spacecraft mass. We work in the non-inertial, co-rotating (synodic) frame of reference, with origin at the center of mass of the Earth-Moon system, in which the Earth and Moon are stationary. The $x$-axis is directed towards the Moon, the $z$-axis is in the direction of the inertial angular velocity of the primary system, and the $y$-axis completes the right-handed $x-y-z$ orthogonal frame as shown in Figure 2 .

The phase coordinates are non-dimensionalized so that the mass, time and length units are respectively the sum of the masses of the primaries, the mean orbital period of the primaries around the center of mass of the system, and the distance between the Earth and the Moon. The mass parameter, $\mu:=$ $m_{2} /\left(m_{1}+m_{2}\right)$, is the only parameter in the resulting dynamical model. For the Earth-Moon system the mass parameter is 0.01215 . The lengths of the position vectors to the secondary mass (spacecraft) from the primary masses are $r_{1}=\sqrt{(x+\mu)^{2}+y^{2}+z^{2}}$ and $r_{2}=\sqrt{(x+\mu-1)^{2}+y^{2}+z^{2}}$ respectively.

The positions and velocities of the secondary mass are the coordinates of the phase point $\mathbf{x}=$ $\left(x, y, z, v_{x}, v_{y}, v_{z}\right)^{T}$. The spacecraft dynamics, written as a system of six first-order ordinary differential equations $(n=6)$, are 


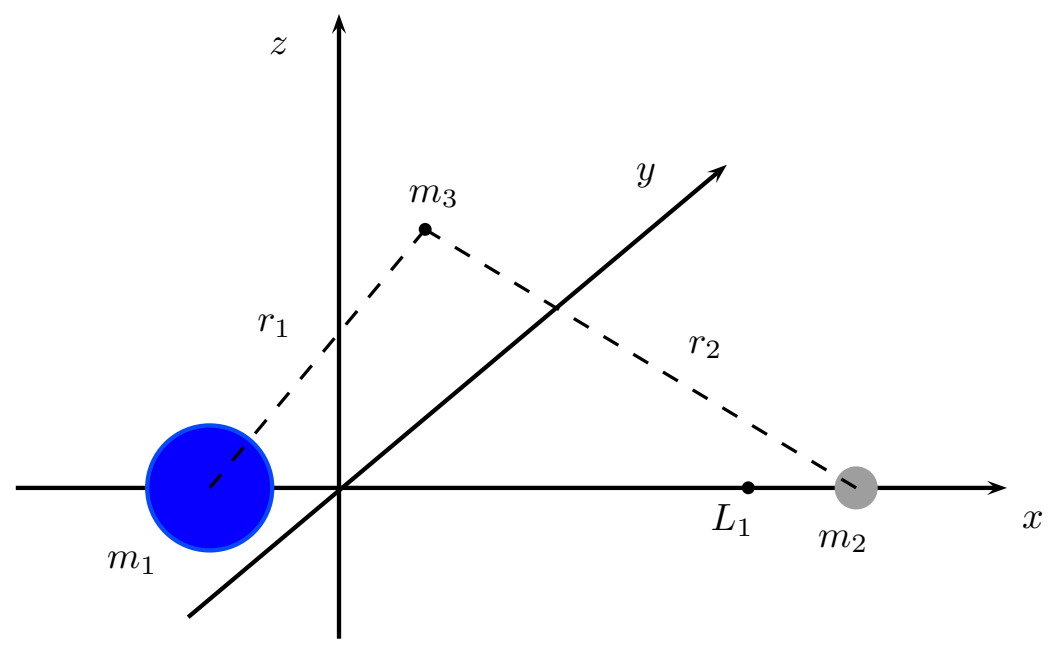

Figure 2. Frame of reference in the Earth-Moon Circular Restricted Three-Body Problem.

$$
\begin{aligned}
\dot{x} & =v_{x}, \\
\dot{y} & =v_{y}, \\
\dot{z} & =v_{z}, \\
\dot{v}_{x} & =x+2 v_{y}-(1-\mu)(x+\mu) r_{1}^{-3}-\mu(x-1+\mu) r_{2}^{-3}, \\
\dot{v}_{y} & =y-2 v_{x}-(1-\mu) y r_{1}^{-3}-\mu y r_{2}^{-3}, \\
\dot{v}_{z} & =-(1-\mu) z r_{1}^{-3}-\mu z r_{2}^{-3} .
\end{aligned}
$$

The system (3.1) has five equilibria at which the spacecraft is stationary in the synodic frame. These libration points, $L_{1}$ through $L_{5}$, all lie in the $x$-y plane. $L_{1}, L_{2}$, and $L_{3}$ are collinear and lie on the $x$-axis, while $L_{4}$ and $L_{5}$ are symmetric with respect to the $x$-axis and each is a vertex of an equilateral triangle with the primaries $m_{1}$ and $m_{2}$ at the other vertices. We focus on a neighborhood of the Earth-Moon $L_{1}$ libration point located between the Earth and the Moon at $(x, y, z)=(0.8369180073,0,0)$ as shown in Figure 2. In the phase space, the $L_{1}$ equilibrium point has a 'saddle $\times$ center $\times$ center' structure with two real eigenvalues equal in magnitude and opposite in sign and two pairs of purely imaginary conjugate eigenvalues. Thus $L_{1}$ is an unstable equilibrium.

\subsection{Finite-Time Partially Hyperbolic Points Near $L_{1}$}

In the two subsections following this one, we investigate 20 points in the phase space neighborhood of $L_{1}$. Here we use $L_{1}$ to refer to the phase point with position coordinates given in the previous subsection and zero velocity coordinates in the synodic frame; whether $L_{1}$ is being considered a position or phase point should be clear from the context. We find that all 20 points are uniform finite-time partially hyperbolic, with forward and backward spectra divided in three subsets of dimension $n^{s}=1, n^{c}=4$, and $n^{u}=1$. Because the system in (3.1) is Hamiltonian, the FTLEs are symmetric with respect to zero.

For the partially hyperbolic spectrum identified, we can construct a splitting of the tangent space (2.10) using the forward and backward FTLVs

$$
T_{\mathbf{x}} \mathbb{R}^{n}=\mathcal{E}^{s}(T, \mathbf{x}) \oplus \mathcal{E}^{c}(T, \mathbf{x}) \oplus \mathcal{E}^{u}(T, \mathbf{x}),
$$

with the subspaces defined by the appropriate FTLVs based on (2.8) and (2.11), namely 


$$
\begin{aligned}
& \mathcal{E}^{s}(T, \mathbf{x})=\operatorname{span}\left\{\mathbf{l}_{1}^{+}(T, \mathbf{x})\right\} \\
& \mathcal{E}^{u}(T, \mathbf{x})=\operatorname{span}\left\{\mathbf{l}_{6}^{-}(T, \mathbf{x})\right\} \\
& \mathcal{E}^{c}(T, \mathbf{x})=\left(\left[\mathcal{E}^{c}(T, \mathbf{x})\right]^{\perp}\right)^{\perp}, \quad\left[\mathcal{E}^{c}(T, \mathbf{x})\right]^{\perp}=\operatorname{span}\left\{\mathbf{l}_{6}^{+}(T, \mathbf{x}), \mathbf{l}_{1}^{-}(T, \mathbf{x})\right\}
\end{aligned}
$$

Although there is some variation in the convergence time constant, $(\Delta \mu)^{-1}$, the values are all less than unity; thus the splittings can be accurately resolved with an averaging time $T$ on the order of 5 .

\subsection{Periodic $L_{1}$ Orbits: Lyapunov Subspaces Compared to Floquet Subspaces}

Nine periodic Lyapunov horizontal orbits and 3 periodic halo orbits around $L_{1}$ are computed using the software AUTO [9]. Figures 3 and 4 show the orbits and the FTLEs as functions of $T$ for the indicated points, one per orbit. All 12 points are uniform finite-time partially hyperbolic with the dimensions noted in the previous subsection, and thus, at each point, a tangent space splitting into stable, center, and unstable subspaces as in the first of equations (3.2) can be computed using FTLA.

For periodic orbits, Floquet theory is applicable and allows a quantitative accuracy assessment of FTLA. Using Floquet theory, the stable, center, and unstable subspaces can be determined from the monodromy matrix. The monodromy matrix $M(\mathbf{x})$, for $\mathbf{x}$ on a periodic orbit, is the transition matrix at $t=T_{p}$ where $T_{p}$ is the orbit period, namely

$$
M(\mathbf{x})=\Phi\left(T_{p}, \mathbf{x}\right) .
$$

The eigenvectors of the monodromy matrix can be used to define stable, center, unstable subspaces of the tangent space and in this case the subspaces are $\Phi$-invariant (to the accuracy of the numerics). The FTLA subspaces would in principle converge asymptotically to the Floquet subspaces; however we will demonstrate that the practical convergence is much quicker.

The Floquet subspaces are compared to the FTLA subspaces for a range of averaging times T. Figure 5 shows the value of the largest principal angles between the stable, center, and unstable subspaces calculated via the two methods. As the averaging time $T$ for FTLA increases, the angles between the Floquet and FTLA subspaces decrease at the expected exponential rates, showing that the FTLA subspaces accurately approximate the invariant subspaces for sufficiently large $T$. The periods for the Lyapunov horizontal orbits are in the interval $[2.7,3.2]$ in nondimensional time. For the 9 points on these orbits, the interval of spectral gaps is $[1.4,2.6]$ and 5 convergence time constants requires averaging times in the interval $[3.1,4.7]$. Thus for these points Floquet analysis requires less integration time, but this is not always the case because the required FTLA averaging time depends on the spectral gap. For a fixed $T$, the angles are larger the more energetic the orbits are. This is because more energetic orbits have smaller spectral gaps $\Delta \mu$, as shown in Figures 3 and 4 , and thus more averaging time is required in order to achieve the same accuracy. Anyway the motivation for FTLA is not to compete with Floquet analysis for periodic orbits, but rather to address aperiodic orbits.

\subsection{Quasi-Periodic $L_{1}$ Orbits: Verifying Lyapunov Subspaces via Simulation}

Mission requirements often do not specify a spacecraft to lie on a particular periodic orbit; instead it is sufficient that the spacecraft trajectory is confined to a neighborhood of a specified periodic orbit. In many cases, associated with a periodic orbit in the CR3BP is a family of quasi-periodic orbits (e.g. quasi-halo and Lissajous orbits) which lie on 2-dimensional tori within the center manifold $\mathcal{W}^{c}$ of $L_{1}$. Each quasi-periodic orbit can be parametrized by two frequencies $\omega_{1}$ and $\omega_{2}$ [33]. We also associate a quasi-periodic orbit with its energy level $E$. The (relative) energy is an integral of the system (3.1), related to the Jacobi integral [32], and can be expressed as

$$
E(\mathbf{x})=\frac{1}{2}\left(v_{x}^{2}+v_{y}^{2}+v_{z}^{2}\right)-\frac{1}{2}\left(x^{2}+y^{2}\right)+\frac{\mu-1}{r_{1}}-\frac{\mu}{r_{2}} .
$$



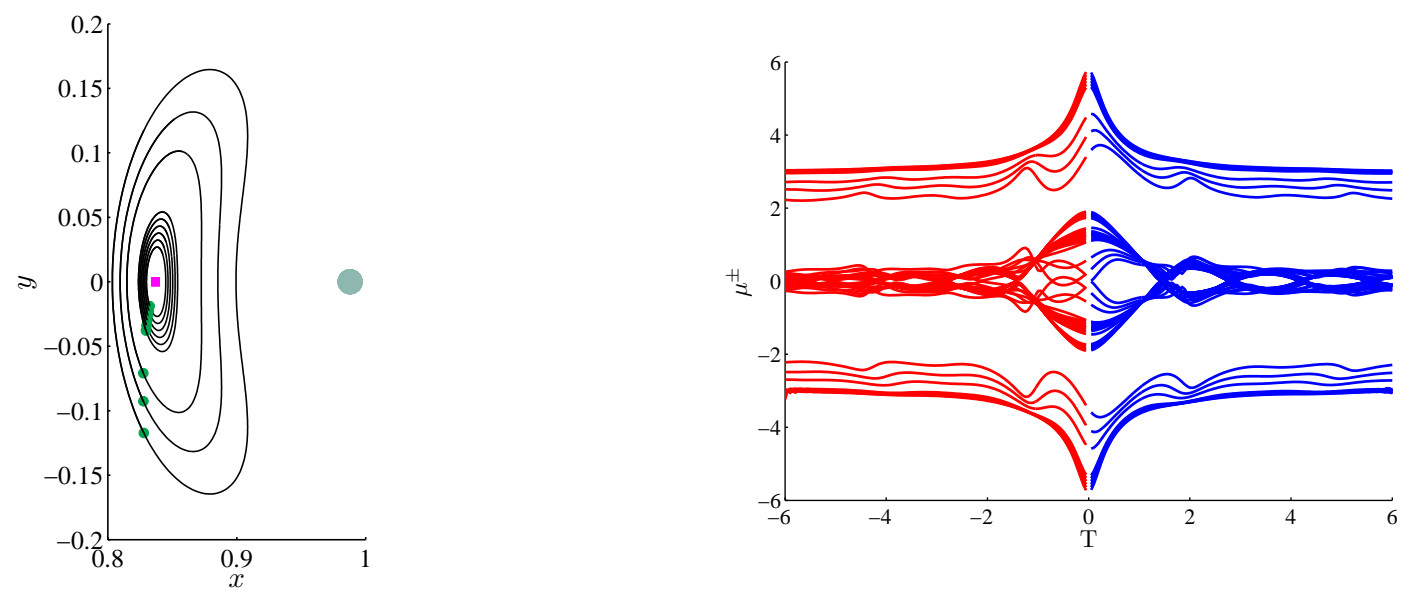

Figure 3. Left: Horizontal Lyapunov orbits around $L_{1}$ (small square). The Moon (solid circle) is also shown. Right: Forward and backward FTLEs versus averaging time for the green points highlighted on the orbits. The spectral gap in the exponents is smaller for the outer, more energetic orbits.
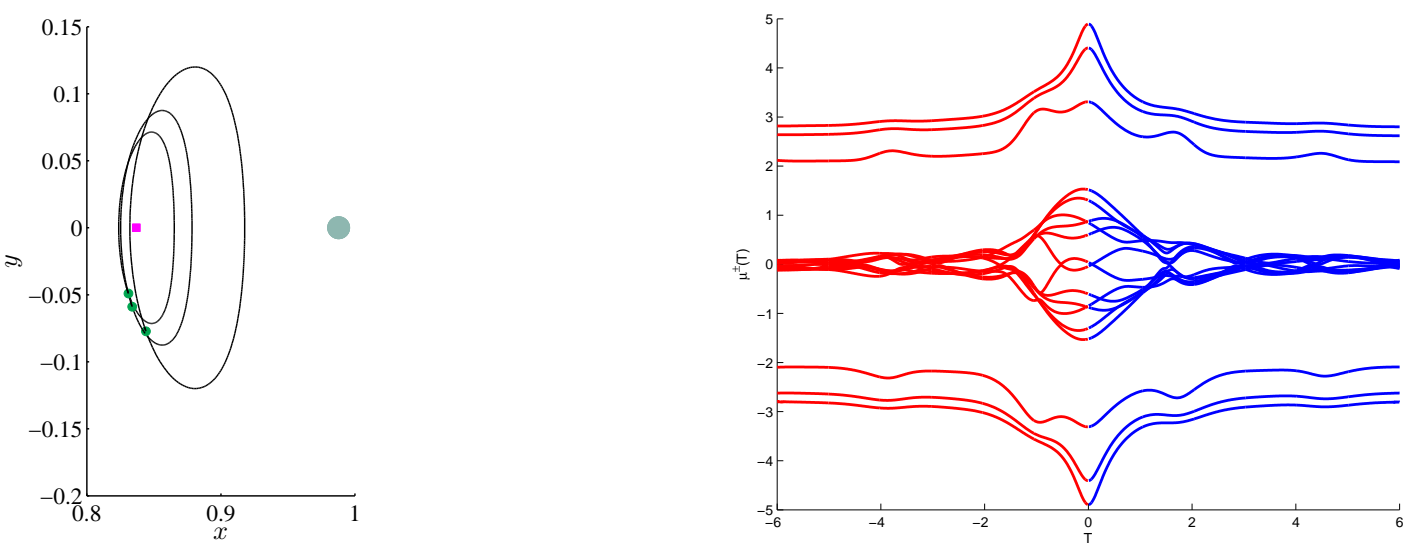

Figure 4. Left: Halo orbits about $L_{1}$ (small square). The Moon (solid circle) is also shown. Right: Forward and backward FTLEs versus averaging time for the green points highlighted on the orbits. The spectral gap in the exponents is smaller for the outer, more energetic orbits.

As mentioned, stationkeeping strategies inspired by dynamical system theory require determining the stable and unstable subspaces $\mathcal{E}^{s}$ and $\mathcal{E}^{u}$ in the tangent space at an orbit point. We will demonstrate via simulation that accurate approximations of the subspaces can be computed by FTLA. To assess the accuracy of the FTLA-derived stable subspace as a local approximation to the associated stable manifold, we monitor the distance between $\phi(t, \mathbf{x})$ and $\phi(t, \mathbf{x}+\delta \mathbf{x})$, where $\mathbf{x}$ is a point on a quasi-periodic orbit and $\delta \mathbf{x} \in \mathcal{E}^{s}(T, \mathbf{x})$, to see if it decreases in forward time, and if so, for what duration of time it decreases. Similarly, to assess the accuracy of the FTLA-derived unstable subspace as a local approximation to the associated unstable manifold, we use a perturbation $\delta \mathbf{x} \in \mathcal{E}^{u}(T, \mathbf{x})$ and monitor the distance in backward time.

Eight quasi-halo orbits are considered with four energy levels and two orbits per energy level; the data were provided by [34]. For each energy level, the two quasi-halo orbits are chosen such that they lie on different tori. One point is selected on each of the 8 orbits. FTLA determines that all 8 points are 

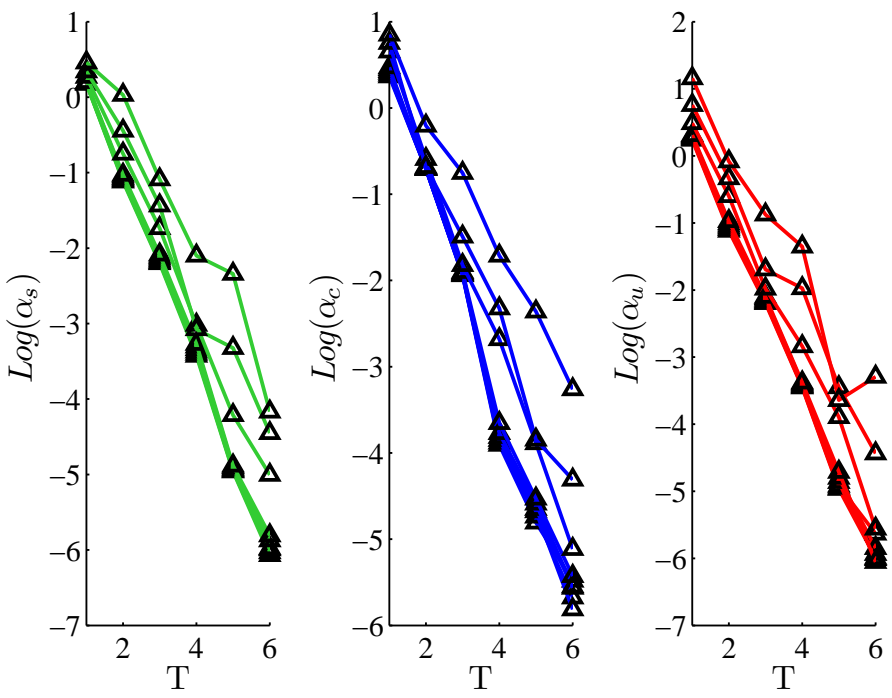

FiguRE 5. Logarithm of angles in radians between subspaces - $\alpha_{s}$ for stable, $\alpha_{c}$ for center, $\alpha_{u}$ for unstable - computed via FTLA and via Floquet theory as a function of the FTLA averaging time $T$. The lines between values (triangles) associate values at different averaging times for the same orbit point.

uniform finite-time partially hyperbolic. Table 1 presents, for each of the 8 points, the coordinates, the characteristics of the corresponding quasi-halo orbit, and the FTLA data: the spectral gap $\Delta \mu$, the start time $t_{s}$, and the number of convergence time constants $\Delta \mu\left(T-t_{s}\right)$ for $T=3.50$. The start time $t_{s}$ is relevant for convergence results and is set by observing when the transient in FTLEs has ended; see right sides of Figures 3 and 4 . The cut-off time $t_{c}$ is zero for all the points; it is only needed for determining the exponential bounds, which are not considered in this example. All the data in Table 1 are nondimensional. At each point, approximations of $\mathcal{E}^{s}$ and $\mathcal{E}^{u}$ are computed as in (3.3) for two averaging times: $T=1.25$ and $T=3.50$.

Figure 6 shows, for point 3, the spatial distance between the corresponding position on the perturbed trajectory and the quasi-periodic orbit as a function of propagation time. We show results only for point 3 because they are representative of the results for all the other points. For both the forward simulation of the perturbation in the FTLA stable subspace and the backward simulation of the perturbation in the FTLA unstable subspace, as the propagation times increases, the spatial distance decreases for some duration and then grows. The reason for this behavior could be the inaccuracy in the FTLA subspaces or the curvature of the stable and unstable manifolds; however, the size of perturbation is small enough that the latter possibility is ruled out. Thus the perturbation in the FTLA stable subspace has a small unstable component that grows exponentially in forward time and eventually causes the distance to grow. Similarly the perturbation in the FTLA unstable subspace has a small stable component that grows exponentially in backward time and eventually causes the distance to grow. The available averaging time for all the points is $\bar{T}=6.0$. Because the spectral gap is $\Delta \mu=2.37, \bar{T}=6.0$ corresponds to about 11 time constants, and smaller averaging times can still provide accurate subspace approximations. Figure 6 shows the cases where $T=1.25$ ( $<1$ time constant) and $T=3.50$ (5.5 time constants) are used. The dashed curves are for $T=1.25$, while the solid curves refer to the case with $T=3.50$. For $T=1.25$ the FTLA subspaces are not as accurate as they are for $T=3.50$ and the distance starts to grow sooner.

For the accuracy assessment of the FTLA subspaces just presented, the total propagation time is similar to the period of the periodic halo orbit associated with the quasi-halo orbit, roughly $T_{p}=2 \pi / \omega_{1}=2.76$ which when dimensionalized is 12 days. Using $T=3.50$ (nondimensional time), FTLA determines the stable and unstable subspaces accurately enough to achieve $7-8$ days of approach towards the reference 
TABLE 1. Points on quasi-halo orbits, orbit parameters, and FTLA parameters for $T=3.50$.

\begin{tabular}{ccccccc} 
& $x$ & $y$ & $z$ & $v_{x}$ & $v_{y}$ & $v_{z}$ \\
\hline 1 & $8.533857 \mathrm{e}-01$ & $-5.998530 \mathrm{e}-02$ & $-2.960509 \mathrm{e}-03$ & $-3.269101 \mathrm{e}-02$ & $-6.243649 \mathrm{e}-02$ & $7.550298 \mathrm{e}-02$ \\
2 & $8.607849 \mathrm{e}-01$ & $-4.275194 \mathrm{e}-02$ & $-9.427094 \mathrm{e}-03$ & $-1.207843 \mathrm{e}-02$ & $-1.432303 \mathrm{e}-01$ & $-1.018362 \mathrm{e}-03$ \\
3 & $8.575485 \mathrm{e}-01$ & $-6.160232 \mathrm{e}-02$ & $-2.301536 \mathrm{e}-02$ & $-3.647974 \mathrm{e}-02$ & $-7.703128 \mathrm{e}-02$ & $8.629800 \mathrm{e}-02$ \\
4 & $8.647505 \mathrm{e}-01$ & $4.838973 \mathrm{e}-02$ & $-2.453995 \mathrm{e}-02$ & $1.253966 \mathrm{e}-02$ & $-1.521651 \mathrm{e}-01$ & $9.960893 \mathrm{e}-05$ \\
5 & $8.677123 \mathrm{e}-01$ & $-4.543023 \mathrm{e}-02$ & $-2.991545 \mathrm{e}-02$ & $-1.930001 \mathrm{e}-02$ & $-1.601081 \mathrm{e}-01$ & $8.183028 \mathrm{e}-02$ \\
6 & $8.665880 \mathrm{e}-01$ & $2.739575 \mathrm{e}-02$ & $-2.376710 \mathrm{e}-02$ & $1.490864 \mathrm{e}-02$ & $-1.717655 \mathrm{e}-01$ & $-1.126149 \mathrm{e}-01$ \\
7 & $8.744849 \mathrm{e}-01$ & $-3.104252 \mathrm{e}-02$ & $-5.494215 \mathrm{e}-02$ & $-1.305636 \mathrm{e}-02$ & $-1.881380 \mathrm{e}-01$ & $4.475706 \mathrm{e}-02$ \\
8 & $8.578558 \mathrm{e}-01$ & $9.324278 \mathrm{e}-02$ & $-2.435399 \mathrm{e}-02$ & $6.819778 \mathrm{e}-02$ & $-1.358021 \mathrm{e}-02$ & $-9.454421 \mathrm{e}-02$ \\
\hline
\end{tabular}

\begin{tabular}{ccccccc} 
& $E$ & $\omega_{1}$ & $\omega_{2}$ & $\Delta \mu$ & $t_{s}$ & $\Delta \mu\left(T-t_{s}\right)$ \\
\hline 1 & -1.5817 & 2.2821 & 0.0935 & 2.46 & 1.2 & 5.7 \\
2 & -1.5817 & 2.2666 & 0.0462 & 2.43 & 1.2 & 5.6 \\
3 & -1.5781 & 2.2776 & 0.1339 & 2.37 & 1.2 & 5.5 \\
4 & -1.5781 & 2.2587 & 0.0887 & 2.37 & 1.2 & 5.5 \\
5 & -1.5739 & 2.2725 & 0.1797 & 2.37 & 1.2 & 5.5 \\
6 & -1.5739 & 2.2513 & 0.1356 & 2.33 & 1.2 & 5.4 \\
7 & -1.5691 & 2.2672 & 0.2317 & 2.26 & 1.4 & 4.7 \\
8 & -1.5691 & 2.2443 & 0.1879 & 2.20 & 1.3 & 4.8 \\
\hline
\end{tabular}

quasi-periodic orbit while the perturbed trajectory shadows the stable manifold for that orbit. After 12 days, the distance is still less than half of what it was initially. The unstable FTLE is 2.8; converting the associated time constant to units of days, we find that the unstable mode grows by a factor of $e$ every 1.55 days.
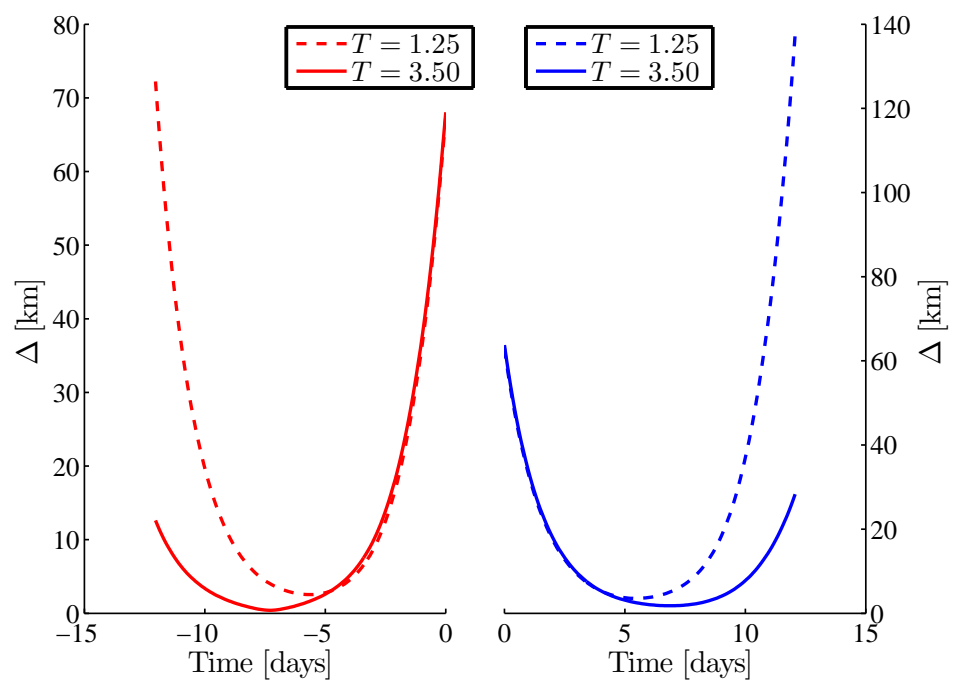

FiguRE 6. Spatial distance between a nominal quasi-halo orbit (starting from point 3 of Table 1) and the trajectories when perturbed along the unstable (left plot) and along the stable (right plot) directions. The curves are obtained by using two different averaging times: $T=1.25$ dashed line and $T=3.5$ solid line. 


\section{Conclusions}

Finite-time Lyapunov exponents, vectors, and subspaces provide a means of analyzing and characterizing geometric structure in the flow of a nonlinear dynamical system. In particular, finite-time Lyapunov analysis of the tangent linear dynamics determines the tangent space structure at phase space points. We have focused on partially hyperbolic structure with a tangent space splitting into stable, center and unstable subspaces. Although exact results require asymptotic limits and either uniform or recurrent behavior, the exponential convergence of the finite-time Lyapunov subspaces toward fixed subspaces makes accurate characterization feasible in finite time.

Finite-time Lyapunov analysis was applied to the dynamics of a spacecraft in a libration point orbit in the circular restricted three-body problem. It was demonstrated that accurate information on stable and unstable directions relevant to stationkeeping can be obtained. For periodic orbits, the information from finite-time Lyapunov analysis is consistent with that from Floquet analysis, provided a sufficiently long averaging time is used for the former. The benefit of Lyapunov analysis is that it is not limited to periodic orbits. It was demonstrated by simulation that the stable and unstable tangent subspaces at a point on a quasi-periodic orbit can be accurately determined.

Acknowledgements. This material is based upon work supported by the National Science Foundation under Grant CMMI-1069331.

\section{References}

[1] A. Adrover, F. Creta, M. Giona, M. Valorani, V. Vitacolonna. Natural Tangent Dynamics with Recurrent Biorthonormalization: A Geometric Computational Approach to Dynamical Systems Exhibiting Slow Manifolds and Periodic/Chaotic Limit Sets. Physica D, (2) 213 (2006), 121-146.

[2] E.M. Alessi, G. Gómez, J.J. Masdemont. Leaving The Moon by Means of Invariant Manifolds of Libration Point Orbits, Commun. Nonlinear Sci. Numer. Simulat., 14 (2009), 4153-4167.

[3] L. Barreira, Y.B. Pesin. Lyapunov Exponents and Smooth Ergodic Theory. University Lecture Series 23. American Mathematical Society, Providence, 2002.

[4] M. Belló, G. Gómez, J.J. Masdemont. Invariant Manifolds, Lagrangian Trajectories and Space Mission Design. In: Space Manifold Dynamics. Springer, 2010, 1-96.

[5] M. Branicki, A. M. Macho, S. Wiggins. A Lagrangian Description of Transport Associated with a Front-Eddy Interaction: Application to Data from the North-Western Mediterranean Sea. Physica D, 240 (2011), $282-304$.

[6] E. Chiavazzo, A.N. Gorban, I.V. Karlin. Comparison of Invariant Manifolds for Model Reduction in Chemical Kinetics. Comm. in Comp. Physics, 2 (5) (2007), 964-992.

[7] L. Dieci, E.S. Van Vleck. Lyapunov Spectral Intervals: Theory and Computation. SIAM J. Numerical Analysis, 40 (2) (2002), 516-542.

[8] E.J. Doedel, V.A. Romanov, R.C. Paffenroth, H.B. Keller, D.J. Dichmann, J. Galán-Vioque, A. Vanderbauwhede. Elemental Periodic Orbits associated with the Libration Points in the Circular Restricted 3-Body Problem. International Journal of Bifurcation and Chaos, 17 (8) (2007), 2625-2677.

[9] E.J. Doedel, AUTO, ftp://ftp.cs.concordia.ca/pub/doedel/auto.

[10] D.C. Folta, T.A. Pavlak, A.F. Haapala, K.C. Howell, M.A. Woodard. Earth-Moon Libration Point Stationkeeping: Theory, Modeling, and Operations. Acta Astronautica, 94 (1) (2014), 421-433.

[11] C. Froeschle, E. Lega, R. Gonczi. Fast Lyapunov Indicators: Application to Asteroidal Motion. Celestial Mechanics and Dynamical Astronomy, 67 (1997), 41-62.

[12] C.W. Gear, T.J. Kaper, I.G. Kevrekidis, A. Zagaris. Projecting to a Slow Manifold: Singularly Perturbed Systems and Legacy Codes. SIAM J. Appl. Dyn. Syst., 4 (2005), 711-732.

[13] G.H. Golub, C.F. Van Loan. Matrix Computations, 3rd Edition. The Johns Hopkins University Press, Baltimore, 1996.

[14] G. Gómez, J. Llibre, R. Martínez, C. Simó. Station Keeping of Libration Point Orbits. Technical Report ESOC Contract $5648 / 83 / \mathrm{D} / \mathrm{JS}(\mathrm{SC}), 1985$.

[15] G. Gómez, J. Masdemont, C. Simó. Study of the Transfer from the Earth to a Halo Orbit Around the Equilibrium Point L1. Celestial Mechanics and Dynamical Astronomy, 56 (1993), 541-562.

[16] G. Haller. Finding Finite-Time Invariant Manifolds in Two-Dimensional Velocity Fields. Chaos, 10 (1) (2000), $99-108$.

[17] B. Hasselblatt, Y.B. Pesin. Partially Hyperbolic Dynamical Systems. In: B. Haselblatt and A. Katok (Eds.), Handbook of Dynamical Systems. vol. 1B. Elsevier, New York, 2005.

[18] K.C. Howell, B. Barden, M. Lo. Application of Dynamical Systems Theory to Trajectory Design for a Libration Point Mission. Journal of Astronautics Science, 45 (2) (1997), 161-178.

[19] T.M. Keeter. Station-Keeping Strategies for Libration Point Orbits: Target Point and Floquet Mode Approaches. Ph.D. Dissertation. Purdue University, 1994. 
[20] W.S. Koon, M.W. Lo, J.E. Marsden, S.D. Ross. Heteroclinic Connections between Periodic Orbits and Resonance Transitions in Celestial Mechanics. Chaos, 10 (2) (2000), 427-469.

[21] S.H. Lam, D.A. Goussis. The CSP Method for Simplifying Kinetics. Int. J. Chemical Kinetics, 26 (1994), $461-486$.

[22] U. Maas, S.B. Pope. Simplifying Chemical Kinetics: Intrinsic Low-Dimensional Manifolds in Composition Space. Combustion and Flame, 88 (1992), 239-264.

[23] K.D. Mease. Multiple Timescales in Nonlinear Flight Mechanics: Diagnosis and Modeling. Applied Mathematics and Computation, 164 (2005), 627-648.

[24] K.D. Mease, U. Topcu, E. Aykutluğ, M. Maggia. Characterizing Two-Timescale Nonlinear Dynamics Using FiniteTime Lyapunov Exponents and Vectors. (2014). arXiv:0807.0239v3 [math.DS].

[25] D.S. Naidu, A.J. Calise. Singular Perturbations and Timescales in Guidance and Control of Aerospace Systems: a Survey,. J. Guidance, Control and Dynamics, 24 (6) (2001), 1057-1078.

[26] R.E. O'Malley. Singular Perturbation Methods for Ordinary Differential Equations. Springer-Verlag, New York, 1991.

[27] M.R. Roussel, S.J. Fraser. Geometry of the Steady-State Approximation: Perturbation and Accelerated Convergence Methods. J. Chem. Phys., 93 (3) (1990), 1072-1081.

[28] L.A. Segel, M. Slemrod. The Quasi-Steady-State Assumption: a Case Study in Perturbation. SIAM Review, 31 (3) (1989), 446-477.

[29] S. C. Shadden, F. Lekien, J. E. Marsden, Definition and Properties of Lagrangian Coherent Structures from FiniteTime Lyapunov Exponents in Two-Dimensional Aperiodic Flows. Physica D, 212 (2005), 271-304.

[30] C.R. Short, K.C. Howell. Flow Control Segment and Lagrangian Coherent Structure Approaches for Application in Multi-Body Problems. Acta Astronautica 94 (2014), 562-607.

[31] C. Simó, G. Gómez, J. Llibre, R. Martínez. Station Keeping of a Quasiperiodic Halo Orbit Using Invariant Manifolds. In: Proceedings of the Second International Symposium on Spacecraft Flight Dynamics. Darmstadt, FR Germany, 20-23 October, 1986 (ESA SP-255, Dec. 1986).

[32] V. Szebehely. Theory of Orbits: The Restricted Problem of Three Bodies. Academic Press, New York, 1967.

[33] H. Wan, B.F. Villac. Lunar L1 Earth-Moon Propellant Depot Orbital and Transfer Options Analysis. AAS 13-885, (2013).

[34] H. Wan, personal communication, 2013.

[35] S. Yoden, M. Nomura. Finite-Time Lyapunov Stability Analysis and Its Application to Atmospheric Predictability. J. Atmospheric Sciences, 50 (11) (1993), 1531-1543. 